\title{
ORIGINAL
}

\section{COMPARACIÓN DE LOS MODELOS SCORE Y REGICOR PARA EL CÁLCULO DEL RIESGO CARDIOVASCULAR EN SUJETOS SIN ENFERMEDAD CARDIOVASCULAR ATENDIDOS EN UN CENTRO DE SALUD DE BARCELONA}

José Miguel Baena Díez (1,2), José Luis del Val García (2), Luis Héctor Salas Gaetgens (1), Ricard Sánchez Pérez (1), Eva Altes Vaques (1), Bibiana Deixens Martínez (1), Marta Amatller Corominas (1) y Dynaida Katia Núñez Casillas (1)

(1) Área Básica de Salud (ABS) Dr. Carles Ribas-La Marina (Barcelona)

(2) UI SAP Sants-Montjuïc-Sarrià-Les Corts-Sant Gervasi

\section{RESUMEN}

Fundamento: No se dispone de estudios que hayan comparado SCORE, REGICOR y Framingham. El objetivo de este trabajo es estudiar cómo clasifican el riesgo cardiovascular las funciones REGICOR y SCORE, su correlación y concordancia respecto a Framingham (1998) y si presentan diferencias respecto a los factores de riesgo cardiovascular en los casos de riesgo alto.

Métodos: Estudio descriptivo transversal realizado en atención primaria. Se incluyó a 851 personas entre 35-74 años, libres de enfermedades cardiovasculares y seleccionadas por muestreo aleatorio simple. Se estudió la proporción de pacientes de riesgo alto con Framingham ( $\geq 20 \%$ a 10 años), SCORE ( $\geq 5 \%$ a 10 años) y REGICOR con puntos de corte $\geq 20 \%, \geq 15 \%, \geq 10 \%$ y $\geq 5 \%$ a 10 años, ya que con REGICOR $\geq 20 \%$ apenas hay casos con riesgo alto. Se comparó la correlación ( $\mathrm{r}$ de Pearson) y concordancia (coeficiente Kappa) de las personas de riesgo alto de REGICOR y SCORE respecto a Framingham.

Resultados: Presentaron riesgo alto 23,3\% con Framingham, $15,2 \%$ con SCORE y un $1,4 \%, 5,8 \%, 17,6 \%$ y $57,0 \%$ con REGICOR con los puntos de corte descritos, respectivamente. REGICOR tuvo una correlación de 0,99 y SCORE de 0,78 . REGICOR $\geq 10 \%$ tuvo mejor concordancia (Kappa 0,83) que SCORE (Kappa 0,61). Al comparar los factores de riesgo cardiovascular de los casos con riesgo alto ( $\geq 20 \%$ Framingham, $\geq 5 \%$ SCORE $y \geq 10 \%$ REGICOR), el segundo presentó mayor prevalencia de diabetes y menor de hipercolesterolemia $(\mathrm{p}<0,05)$

Conclusiones: REGICOR presentó una buena correlación con Framingham. Con el punto de corte $\geq 10 \%$ clasifica como riesgo alto a un número de personas similar a SCORE e inferior a Framingham. El modelo SCORE trataría con hipolipemiantes a un número parecido de pacientes que el modelo REGICOR $\geq 10 \%$, pero con menor evidencia de efectividad del tratamiento.

Palabras clave: Estudios de Validación [Tipo de Publicación]. Factores de riesgo. Enfermedades cardiovasculares.

Correspondencia:

José Miguel Baena Díez.

C. Sant Joan n ${ }^{\circ} 123$, Escalera 2, $3^{\circ} 2^{a}$, Parets del Vallès (08150),

Barcelona

Tel/Fax: 932230549

Correo electrónico: jbaenad@meditex.es
ABSTRACT

\section{Comparison of the SCORE and} REGICOR Models for Calculating Cardiovascular Risk in Cardiovascular Disease-Free Individuals at a Healthcare Center in Barcelona, Spain

Background: No studies have been published to date comparing SCORE, REGICOR and Framingham models. This study is aimed at analyzing how the REGICOR and SCORE functions classify cardiovascular risk, their correlation and concordance with Framingham (1998) and whether any differences exist among them with regard to the cardiovascular risk factors in high-risk groups.

Methods: Descriptive cross-sectional study conducted in primary care. A total of 851 individuals within the 35-74 age range, free of cardiovascular diseases and selected by simple random sampling were included. A study was made of the percentage of high-risk patients with Framingham ( $\geq 20 \%$ ten-year risk), SCORE ( $\geq 5 \%$ tenyear risk) and REGICOR with cutoff points $\geq 20 \%, \geq 15 \%, \geq 10 \%$ and $\geq 5 \%$ at 10 years, given that with REGICOR $\geq 20 \%$ there are hardly any high-risk cases. A comparison was drawn between the correlation (Pearson's r) and concordance (Kappa index) of the REGICOR and SCORE high-risk individuals as compared to Framingham.

Results: The high-risk percentages respectively found were: $23.3 \%$ with Framingham; $15.2 \%$; with SCORE; and $1.4 \%, 5.8 \%$, $17.6 \%$ and $57.0 \%$ with REGICOR with the cutoff points described. REGICOR has a 0.99 correlation, SCORE a 0.78 correlation. REGICOR $\geq 10 \%$ showed a better concordance (Kappa 0.83) than SCORE (Kappa 0.61). On comparing the cardiovascular risk factors of the high-risk cases $(\geq 20 \%$ Framingham, $\geq 5 \%$ SCORE and $\geq 10 \%$ REGICOR), SCORE showed higher prevalence of diabetes and a lower prevalence of hypercholesterolemia $(\mathrm{p}<0.05)$.

Conclusions: REGICOR showed a good correlation with Framingham. With the $>10 \%$ cutoff point, it classifies a number of individuals as high-risk similar to SCORE and fewer than Framingham. The SCORE model would treat a number of patients similar to the REGICOR $\geq 10 \%$ model with hypolipemiant drugs, however showing lesser evidence of effectiveness of the treatment.

Key words: Risk factors. Cardiovascular disease. Validation Studies [Publication Type]. 


\section{INTRODUCCIÓN}

Es conocida la sobreestimación del riesgo cardiovascular (RCV) de las ecuaciones de Framingham en nuestro país ${ }^{1}$. Para subsanarla tenemos actualmente dos alternativas: la calibración realizada por el grupo REGI$\mathrm{COR}^{2}$ y la ecuación del proyecto $\mathrm{SCORE}^{3}$. Sin embargo, no conocemos bien como clasifican el RCV en nuestro medio estas funciones, tanto en el caso de las ecuaciones de Framingham ${ }^{4,5}$ como en las alternativas referidas $^{2,3}$ y sólo 2 trabajos ${ }^{6,7}$ han estudiado algunos aspectos. El primero de ellos ${ }^{6}$ ha comparado la función de Framingham ${ }^{5}$ de 1998 (Wilson) con REGICOR ${ }^{2}$, con el propósito principal de determinar las diferencias en la clasificación del RCV. El segundo ${ }^{7}$ ha contrastado la función de Framingham ${ }^{4}$ de 1991 (Anderson) con SCORE en su versión para países de bajo riesgo ${ }^{3}$, con el objetivo básico de comparar la concordancia en relación a los pacientes calificados como de riesgo alto (RA). Son trabajos poco comparables: diseños distintos, poblaciones seleccionadas de manera diferente, ecuaciones de Framingham distintas y objetivos no exactamente iguales. Seguimos por ello sin conocer cómo clasifican las ecuaciones referidas $^{2,3}$ en una misma población y respecto a una misma función de Framingham a los sujetos definidos como de RA.

Otro aspecto interesante y sujeto a controversias es que con REGICOR ${ }^{2}$ con el punto de corte $\geq 20 \%$ prácticamente no selecciona pacientes de $\mathrm{RA}^{6}$. Apenas se intensificarían las intervenciones para disminuir el RCV y se limitaría la utilidad práctica de la ecuación. Sería por tanto relevante conocer la proporción de pacientes de RA con puntos inferiores de corte, sólo analizada en un estudio $^{6}$.

Puesto que una parte importante de los eventos cardiovasculares se producen en los sujetos mayores de 65 años ${ }^{8}$ es importante conocer el comportamiento de SCORE entre las personas del grupo de edad entre 66 y 74 años, no contempladas en la función original $^{3}$. En el trabajo reseñado ${ }^{7}$ se incluyó a los pacientes hasta los 74 años, asumiendo que el RCV entre los 66-75 años era igual que a los 65 años, premisa difícil de entender, ya que en las ecuaciones de Framingham ${ }^{4,5}$ y REGICOR $^{2}$ el RCV sigue aumentando, sobre todo en varones, entre los 66 y 74 años.

También se ha sugerido que las funciones de Framingham ${ }^{4}$ y $\mathrm{SCORE}^{3}$ podrían clasificar a los pacientes de RA con un diferente perfil ${ }^{7}$ de factores de riesgo cardiovascular (FRCV). Sería interesante comprobar dicha hipótesis y hacerlo también con la función calibrada REGICOR ${ }^{2}$.

Por ello, nuestro objetivo principal fue determinar cómo clasifican las funciones objeto del estudio ${ }^{2,3,5}$ a los pacientes de RA. Como objetivos secundarios comparar la correlación y la concordancia de REGICOR2 y SCORE ${ }^{3}$ con la ecuación de Framingham de 19985, estudiar la proporción de pacientes definidos como RA con distintos puntos de corte con REGICOR ${ }^{2}$, comprobar si con $\mathrm{SCORE}^{3}$ se pueden extrapolar las predicciones entre los 65-74 años ${ }^{7}$ y estudiar si hay diferencias en los casos de RA en relación a los FRCV.

\section{SUJETOS Y MÉTODOS}

\section{Características de la población y selección de los participantes}

Se realizó un estudio descriptivo transversal, realizado en el Centro de Salud Dr. Carles Ribas-la Marina de la ciudad de Barcelona, que lleva funcionando15 años, dentro de una línea de investigación del centro sobre el $\mathrm{RCV}^{9}$. Tenía 35.277 historias clínicas en el momento de realizar el estudio (junio 1998junio 1999). El centro de salud se caracteriza por tener una población adscrita con un nivel socioeconómico heterogéneo, elevada frecuentación, ausencia de hospitales y otros servicios sanitarios relevantes en su área de 
cobertura y población asignada prácticamente igual a la censada.

Los participantes fueron seleccionados por muestreo aleatorio simple a partir del archivo de historias clínicas, con edades entre 35 y 74 años, entre las que se puede calcular el riesgo con REGICOR ${ }^{2}$ y Framingham ${ }^{5}$. En el caso de SCORE se estudió a los pacientes entre los 40 y 74 años. Las personas seleccionadas debían tener registrados los datos basales para poder calcular el riesgo con la funciones ${ }^{2,3,5}$ y estar libres de enfermedades cardiovasculares (cardiopatía isquémica, enfermedad cerebrovascular, arteriopatia periférica de las extremidades inferiores e insuficiencia cardiaca).

\section{Variables de estudio y recogida de los datos}

Se estudiaron las siguientes variables, incluidas como $\mathrm{FRCV}^{2,3,5}$ : la edad, el sexo, el tabaquismo: se consideró como fumador al consumidor diario de cualquier cantidad de tabaco y como exfumador a aquéllos con menos de un año de abstinencia ${ }^{10}$; tensión arterial sistólica y diastólica $(\mathrm{mmHg})$. Se estudió también si estaban diagnosticados ${ }^{11}$ de hipertensión arterial ( 3 tomas $\geq 140 / 90$ $\mathrm{mmHg}$; e) colesterol total (mg/dl). También se determinó si tenían criterios 11 de hipercolesterolemia ( 2 valores $\geq 250 \mathrm{mg} / \mathrm{dl}$ ); f) colesterol HDL (mg/dl); g) diabetes mellitus. Se utilizaron los criterios de la Asociación Americana de Diabetes ${ }^{12}$; RCV a 10 años con REGICOR ${ }^{2}$, SCORE $^{3}$ y Framingham ${ }^{5}$ mediante las ecuaciones originales utilizando la función de sintaxis del programa SPSS.

Para el cálculo del RCV con SCORE se utilizó sólo el colesterol total, ya que según los autores ${ }^{3}$ no hay ventajas al utilizar el HDL. Se calculó el RCV ${ }^{3}$ entre 40-65 años (función original), y entre 40-74 años, puesto que la sintaxis introduce los valores de edad en años, para verificar una de las hipótesis del estudio.
Los datos se recogieron mediante un protocolo estandarizado, precedido de una prueba piloto con 20 personas para detectar errores del diseño y entrenar a los encuestadores. Fueron obtenidos por revisión de la historia clínica y contacto con el médico responsable y entrevista con la persona participante para completar los datos basales. La metodología pormenorizada y el cálculo del tamaño muestral se detallan en el artículo mencionado $^{9}$. Se realizó un control de calidad por dos revisores en 135 protocolos, consistente en comprobar si las variables (cuantitativas y cualitativas) del estudio se habían registrado correctamente.

Se estudió cómo clasifican a los pacientes definidos como RA a 10 años las funciones estudiadas con los siguientes niveles de RCV: $\geq 20 \%$ con Framingham ${ }^{5}$ y REGICOR $^{2}$ y $\geq 5 \%$ con SCORE $^{3}$. La proporción de pacientes de RA a los 10 años con REGICOR se valoró con los siguientes puntos de corte: $\geq 5 \%, \geq 10 \%, \geq 15 \%$ y $\geq 20 \%$, para verificar una de las hipótesis del estudio.

\section{Análisis estadístico}

Se realizó mediante el paquete SPSS, versión 11.0. Dicho paquete también fue utilizado para calcular el RCV con las funciones estudiadas $^{2,3,5}$. El control de calidad se verificó mediante el coeficiente Kappa y el coeficiente de correlación intraclase, considerándose que valores superiores a 0,75 eran indicativos de una buena concordancia. Las proporciones se compararon mediante la prueba de ji al cuadrado y las medias mediante la t de Student y la ANOVA o las pruebas correspondientes si no se cumplían sus condiciones de aplicación. La correlación y concordancia de las funciones estudiadas se analizó mediante la r de Pearson y el coeficiente Kappa, respectivamente. Se adoptó un nivel alfa de significación estadística $<0,05$ en todos los casos. 


\section{RESULTADOS}

La selección inicial fue de 1.124 pacientes, disponiéndose de todos los datos para calcular el riesgo en 851 sujetos $(75,7 \%)$ con una edad media de 55,9 años (DE 11,0; rango: 35-74), de los cuales 496 (58,3\%) eran mujeres. Para el estudio del RCV con SCORE entre 40-65 años se incluyeron 561 casos y con SCORE hasta los 74 años 756 . El control de calidad mostró valores $>0,75$ tanto en el en el coeficiente Kappa como en el coeficiente de correlación intraclase.

En la tabla 1 se detallan los FRCV de los pacientes. En la tabla 2 se reflejan los valores medios de RCV. El RCV medio es más elevado con Framingham y disminuye con REGICOR y SCORE hasta los 65 años. Con SCORE hasta los 74 años los valores medios de RCV fueron similares a REGICOR y casi el doble que con SCORE hasta los 65 años.
La proporción de pacientes con RA (tabla 3 ) fue alta con Framingham $\geq 20 \%$. Con REGICOR prácticamente no existen pacientes de RA con el punto de corte $\geq 20 \%$, aumentado los pacientes con RA al disminuir el punto de corte. Con SCORE hasta los 65 años la proporción fue similar a REGICOR $\geq 10 \%$. Sin embargo, con SCORE hasta 74 años la proporción de pacientes de RA fue superior a Framingham, a expensas sobre todo de las mujeres. En la tabla 4 se detalla la proporción de individuos con diabetes mellitus y RA: SCORE hasta los 65 años presenta casi la misma proporción de pacientes de RA que Framingham, esta proporción es superior con SCORE hasta los 74 años y con REGICOR con el punto de corte $\geq 10 \%$ la proporción es algo inferior a Framingham.

La correlación entre Framingham y REGICOR fue muy elevada (figura 1), con un valor de la r de Pearson de 0,99 (p<0,001). El valor fue de 0,99 en varones y en mujeres. $\mathrm{La}$

Tabla 1

Características de las personas estudiadas en relación a los factores de riesgo, expresadas como media y desviación estándar (entre paréntesis) para las variables cuantitativas y como número y porcentaje (entre paréntesis) para las variables cualitativas

\begin{tabular}{|l|c|c|c|}
\hline & $\begin{array}{c}\text { Varones } \\
\mathbf{n}=\mathbf{3 5 5}\end{array}$ & $\begin{array}{c}\text { Mujeres } \\
\mathbf{n = 4 9 6}\end{array}$ & $\begin{array}{r}\text { Total } \\
\mathbf{n = 8 5 1}\end{array}$ \\
\hline & & & \\
Edad (años) & $56,1(10,9)$ & $55,8(11,1)$ & $55,9(11,0)$ \\
Tabaquismo & $212(59,7 \%)$ & $118(23,8 \%)$ & $330(38,8 \%)$ \\
Colesterol total (mg/dl) & $216,0(37,9)$ & $220,5(37,9)$ & $218,6(37,9)$ \\
Hipercolesterolemia( $\geq 250 \mathrm{mg} / \mathrm{dl})$ & $79(22,3 \%)$ & $139(28,0 \%)$ & $218(25,6 \%)$ \\
Colesterol HDL (mg/dl) & $48,8(11,3)$ & $53,7(12,3)$ & $51,7(12,1)$ \\
Colesterol HDL $<35 \mathrm{mg} / \mathrm{dl}$ & $39(11,0 \%)$ & $20(4,0 \%)$ & $59(6,9 \%)$ \\
Colesterol HDL $>59 \mathrm{mg} / \mathrm{dl}$ & $58(16,4 \%)$ & $150(30,3 \%)$ & $208(24,4 \%)$ \\
Tensión arterial sistólica (mmHg) & $130,1(16,6)$ & $131,9(19,1)$ & $131,2(18,1)$ \\
Tensión arterial diastólica (mmHg) & $79,7(10,1)$ & $80,4(10,6)$ & $80,1(10,4)$ \\
Hipertensión arterial (OMS) & $124(34,9 \%)$ & $200(40,3 \%)$ & $324(38,1 \%)$ \\
Diabetes mellitus & $61(17,2 \%)$ & $63(12,7 \%)$ & $124(14,6 \%)$ \\
\hline
\end{tabular}


Tabla 2

Riesgo medio (expresado como media y DE) de las funciones estudiadas

\begin{tabular}{|l|c|c|c|}
\hline & Varones & Mujeres & Total \\
\hline Riesgo medio Framingham (n=851) & $19,8(12,3)$ & $10,5(7,2)$ & $14,4(10,7)$ \\
Riesgo medio REGICOR ( $\mathrm{n}=851)$ & $7,8(5,0)$ & $5,1(3,3)$ & $6,2(4,3)$ \\
Riesgo medio SCORE 40-65 años (n=561) & $3,7(3,6)$ & $1,7(2,6)$ & $2,5(3,2)$ \\
Riesgo medio SCORE 40-74 años (n=756) & $6,2(7,7)$ & $3,8(6,4)$ & $4,8(7,1)$ \\
\hline
\end{tabular}

Tabla 3

Número y proporción de personas de riesgo alto a los 10 años con las funciones de Framingham, REGICOR y SCORE

\begin{tabular}{|c|c|c|c|}
\hline & Varones & Mujeres & Total \\
\hline Riesgo alto Framingham $\geq 20 \%(\mathrm{n}=851)$ & $147(41,4 \%)$ & $51(10,3 \%)$ & $198(23,3 \%)$ \\
Riesgo alto REGICOR (n=851) & $12(3,4 \%)$ & $0(0 \%)$ & $12(1,4 \%)$ \\
Punto de corte $\geq 20 \%$ & $33(9,3 \%)$ & $16(3,2 \%)$ & $49(5,8 \%)$ \\
Punto de corte $\geq 15 \%$ & $103(29,0 \%)$ & $47(9,5 \%)$ & $150(17,6 \%)$ \\
Punto de corte $\geq 10 \%$ & $249(70,1 \%)$ & $236(47,6 \%)$ & $485(57,0 \%)$ \\
Punto de corte $\geq 5 \%$ & $129(40,7 \%)$ & $95(21,6 \%)$ & $224(29,6 \%)$ \\
\hline Riesgo alto SCORE $(\geq 5 \%)$ & & & \\
\hline Edad 40-65 años $(\mathrm{n}=561)$ & $57(24,3 \%)$ & $28(8,6 \%)$ & $85(15,2 \%)$ \\
\hline
\end{tabular}

correlación de Framingham con SCORE hasta los 65 años fue peor (figura 2), con un valor de $0,78(p<0,001)$, siendo mejor en varones $(0,79)$ que en mujeres $(0,70)$. La correlación de Framingham con SCORE hasta los 74 años (figura 3 ) fue aún más baja, con un valor de 0,70 ( $\mathrm{p}<0,001)$, siendo de nuevo bastante mejor en varones $(0,77)$ que en mujeres $(0,62)$.

La concordancia respecto a los sujetos de RA entre Framingham y REGICOR con distintos puntos de corte se observa en la tabla 5. REGICOR con un riesgo $\geq 10 \%$ con- 
Tabla 4

Número y proporción de personas con diabetes mellitus y riesgo alto a los 10 años con las funciones de Framingham, REGICOR y SCORE

\begin{tabular}{|c|c|c|c|}
\hline & Varones & Mujeres & Total \\
\hline $\begin{array}{l}\text { Riesgo alto Framingham } \geq 20 \%(n=851) \\
\text { Riesgo alto REGICOR }(n=851)\end{array}$ & $48(78,7 \%)$ & $34(54,0 \%)$ & $82(66,1 \%)$ \\
\hline Punto de corte $\geq 20 \%$ & $10(16,4 \%)$ & $0(0 \%)$ & $10(8,1 \%)$ \\
\hline Punto de corte $\geq 15 \%$ & $14(23,0 \%)$ & $13(20,6 \%)$ & $27(21,8 \%)$ \\
\hline Punto de corte $\geq 10 \%$ & $37(60,7 \%)$ & $31(49,2 \%)$ & $68(54,8 \%)$ \\
\hline $\begin{array}{r}\text { Punto de corte } \geq 5 \% \\
\text { Riesgo alto SCORE }(\geq 5 \%)\end{array}$ & $59(96,7 \%)$ & $61(96,8 \%)$ & $120(96,8 \%)$ \\
\hline Edad 40-65 años $(n=561)$ & $24(63,2 \%)$ & $24(68,6 \%)$ & $48(65,8 \%)$ \\
\hline Edad 40-74 años $(n=756)$ & $46(76,7 \%)$ & $52(82,5 \%)$ & $98(79,7 \%)$ \\
\hline
\end{tabular}

Figura 1

Correlación entre el riesgo cardiovascular de Framingham y REGICOR

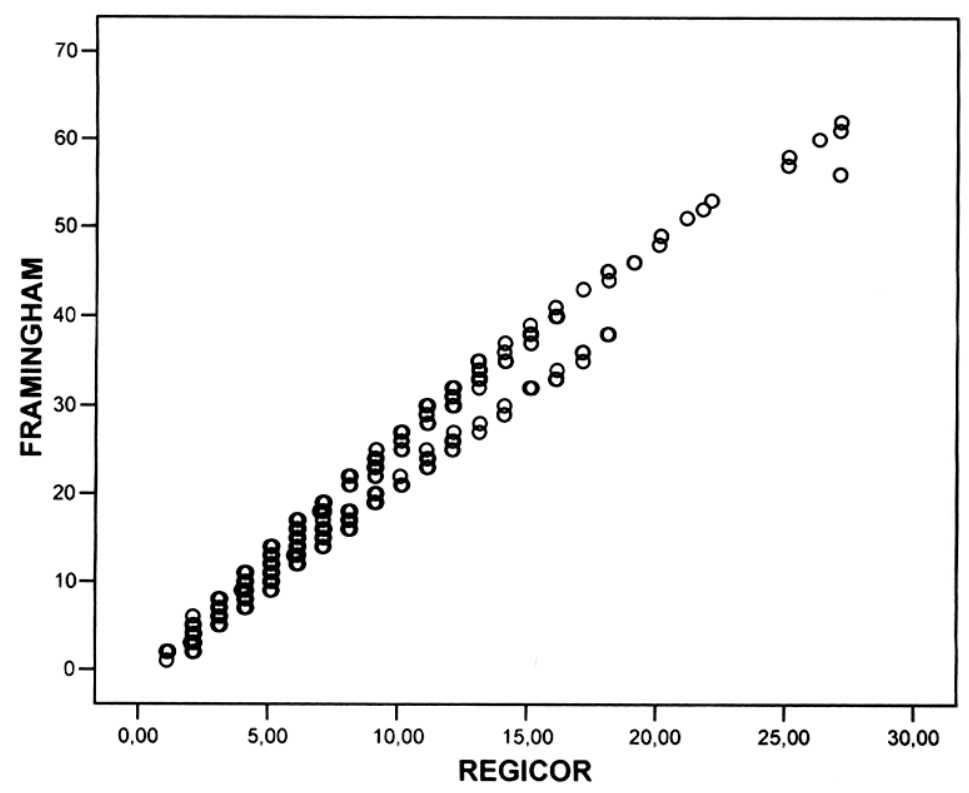


Figura 2

Correlación entre el riesgo cardiovascular de Framingham y SCORE (40-65 años)

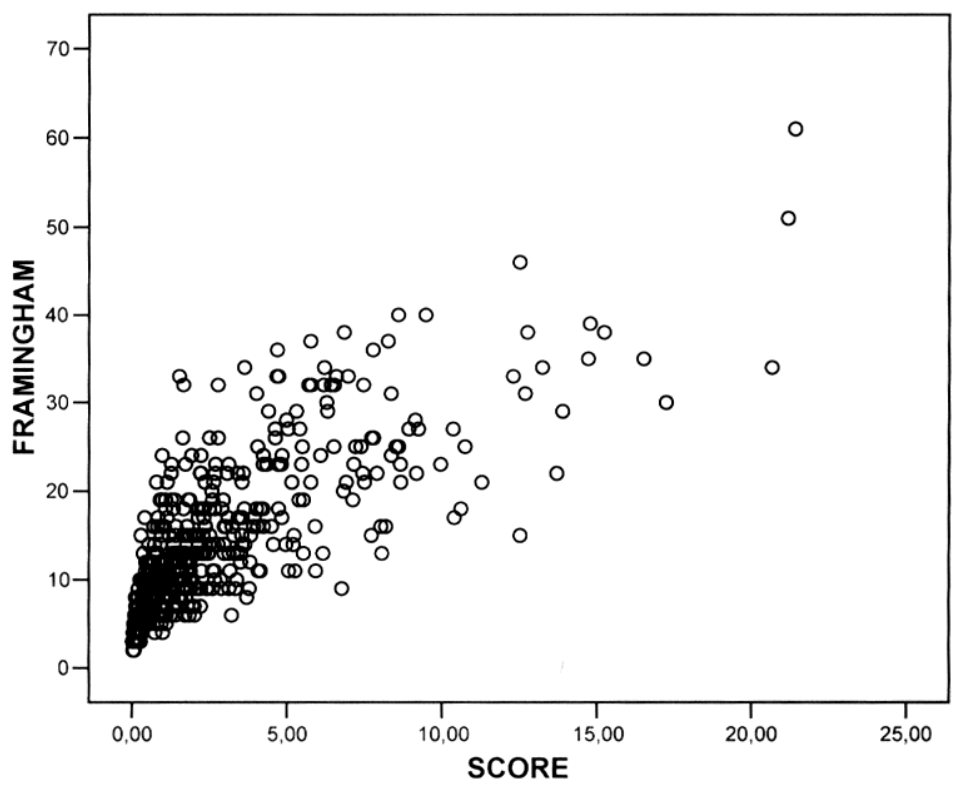

Figura 3

Correlación entre el riesgo cardiovascular de Framingham y SCORE (40-70 años)

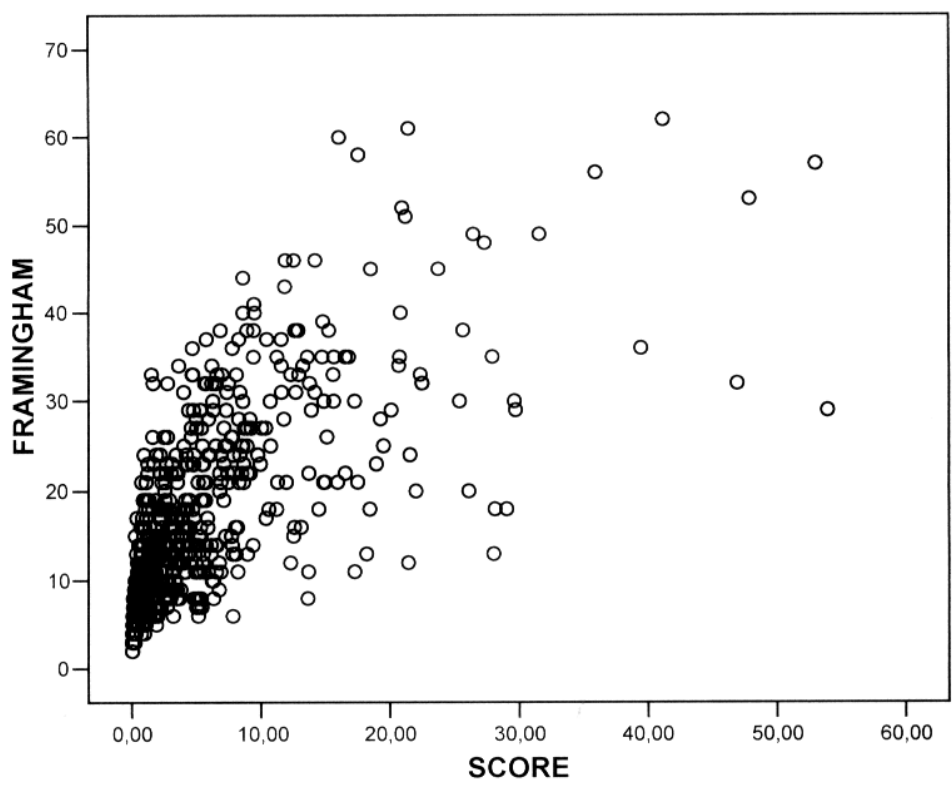


Tabla 5

Concordancia en la clasificación de las personas definidas como de riesgo alto a los 10 años con la ecuación de Framingham (20\%) respecto a REGICOR con distintos puntos de corte. Resultados expresados como número y porcentaje del total

\begin{tabular}{|c|c|c|c|}
\hline Kappa: 0,09 & \multicolumn{2}{|c|}{ REGICOR $\geq 20 \%$} & \multirow[b]{2}{*}{ Total } \\
\hline Framingham & Riesgo bajo & Riesgo Alto & \\
\hline Riesgo bajo & $653(76,7 \%)$ & $0(0 \%)$ & $653(76,7 \%)$ \\
\hline Riesgo alto & $186(21,9 \%)$ & $12(1,4 \%)$ & $198(23,3 \%)$ \\
\hline Total & $839(98,6 \%)$ & $12(1,4 \%)$ & $851(100 \%)$ \\
\hline Kappa: 0,34 & \multicolumn{2}{|c|}{ REGICOR $\geq 15 \%$} & \multirow[b]{2}{*}{ Total } \\
\hline Framingham & Riesgo bajo & Riesgo Alto & \\
\hline Riesgo bajo & $653(76,7 \%)$ & $0(0 \%)$ & $653(76,7 \%)$ \\
\hline Riesgo alto & $149(17,5 \%)$ & $49(5,8 \%)$ & $198(23,3 \%)$ \\
\hline Total & $802(94,2 \%)$ & $49(5,8 \%)$ & $851(100 \%)$ \\
\hline Kappa: 0,83 & \multicolumn{2}{|c|}{ REGICOR $\geq 10 \%$} & \multirow[b]{2}{*}{ Total } \\
\hline Framingham & Riesgo bajo & Riesgo Alto & \\
\hline Riesgo bajo & $653(76,7 \%)$ & $0(0 \%)$ & $653(76,7 \%)$ \\
\hline Riesgo alto & $48(5,6 \%)$ & $150(17,6 \%)$ & $198(23,3 \%)$ \\
\hline Total & $701(82,4 \%)$ & $150(17,6 \%)$ & $851(100 \%)$ \\
\hline Kappa: 0,37 & \multicolumn{2}{|c|}{ REGICOR $\geq 5 \%$} & \multirow[b]{2}{*}{ Total } \\
\hline Framingham & Riesgo bajo & Riesgo Alto & \\
\hline Riesgo bajo & $366(43,0 \%)$ & $287(33,7 \%)$ & $653(76,6 \%)$ \\
\hline Riesgo alto & $0(0 \%)$ & $198(23,3 \%)$ & $198(23,3 \%)$ \\
\hline Total & $366(43,0 \%)$ & $485(57,0 \%)$ & $851(100 \%)$ \\
\hline
\end{tabular}

Tabla 6

Concordancia en la clasificación de las personas definidas de riesgo alto a los 10 años con la ecuación de Framingham ( $\geq 20 \%)$ respecto a SCORE original (hasta los 65 años) y SCORE hasta los 74 años $(\geq 5 \%)$. Resultados expresados como número y porcentaje del total

\begin{tabular}{|c|c|c|c|}
\hline Kappa: 0,61 & \multicolumn{2}{|c|}{ SCORE hasta los 65 años } & \multirow[b]{2}{*}{ Total } \\
\hline Framingham & Riesgo bajo & Riesgo Alto & \\
\hline Riesgo bajo & $434(77,4 \%)$ & $20(3,6 \%)$ & $454(80,9 \%)$ \\
\hline Riesgo alto & $42(7,5 \%)$ & $65(11,6 \%)$ & $107(19,1 \%)$ \\
\hline Total & $476(84,8 \%)$ & $85(15,2 \%)$ & $561(100 \%)$ \\
\hline Kappa: 0,63 & \multicolumn{2}{|c|}{ SCORE hasta los 74 años } & \multirow[b]{2}{*}{ Total } \\
\hline Framingham & Riesgo bajo & Riesgo Alto & \\
\hline Riesgo bajo & $488(64,6 \%)$ & $70(9,3 \%)$ & $558(73,8 \%)$ \\
\hline Riesgo alto & $44(5,8 \%)$ & $154(20,4 \%)$ & $198(26,2 \%)$ \\
\hline Total & $532(70,4 \%)$ & $224(29,6 \%)$ & $756(100 \%)$ \\
\hline
\end{tabular}


Tabla 7

Características en relación a los factores de riesgo cardiovascular de las personas clasificadas de riesgo alto a los 10 años con las funciones estudiadas

\begin{tabular}{|l|c|c|c|}
\hline Factor de riesgo & $\begin{array}{c}\text { Framingham } \geq \mathbf{2 0 \%} \\
\mathbf{n = 1 9 8}\end{array}$ & $\begin{array}{c}\text { REGICOR } \geq \mathbf{1 0 \%} \\
\mathbf{n = 1 5 0}\end{array}$ & $\begin{array}{c}\text { SCORE } \geq \mathbf{5 \%} \\
\mathbf{n = 8 5}\end{array}$ \\
\hline Edad(media y DE) $^{1}$ & $63,9(6,0)$ & $65,0(6,3)$ & $62,0(3,4)$ \\
Sexo masculino(\%) $^{2}$ & $147(74,2 \%)$ & $103(68,7 \%)$ & $57(67,1 \%)$ \\
Tabaquismo(\%) $^{3}$ & $112(56,6 \%)$ & $87(58,0 \%)$ & $47(55,3 \%)$ \\
Hipercolesterolemia(\%) $^{4}$ & $76(38,4 \%)$ & $60(40,0 \%)$ & $21(24,7 \%)$ \\
Hipertensión arterial(\%) $^{5}$ & $133(67,2 \%)$ & $104(69,3 \%)$ & $55(64,7 \%)$ \\
Diabetes mellitus(\%) $^{6}$ & $81(40,9 \%)$ & $65(43,3 \%)$ & $48(56,5 \%)$ \\
\hline
\end{tabular}

${ }^{1} \mathrm{p}<0,001 ;{ }^{2} \mathrm{p}=0,360 ;{ }^{3} \mathrm{p}=0,170 ;{ }^{4} \mathrm{p}=0,044 ;{ }^{5} \mathrm{p}=0,763 ;{ }^{6} \mathrm{p}=0,049$

cuerda muy bien con Framingham, con un kappa de 0,83 (varones 0,73 y mujeres 0,96 ). Las casillas discordantes son sólo el 5,6\% y de los pacientes calificados como de RA por Framingham, REGICOR concuerda en el $75,8 \%$ de los casos. La concordancia es inferior (tabla 6) con SCORE hasta los 65 años, con un kappa de 0,61 (varones 0,63 y mujeres 0,49$)$. Las casillas discordantes aumentan hasta el $11,1 \%$ y de los pacientes de RA con Framingham, SCORE concuerda en el $60,7 \%$. La concordancia con SCORE hasta los 74 años (tabla 6) fue algo mejor, a diferencia del caso anterior con la correlación, con un Kappa de 0,63 (0,72 en varones y 0,44 en mujeres). La proporción de casillas discordantes fue del $15,1 \%$ y concuerdan con Framingham el 77,8\% de los pacientes calificados como de RA.

Por último, en la tabla 7 se estudian los FRCV de los pacientes definidos como RA a los 10 años. Además de la edad (SCORE tiene un diferente rango) se observan 2 diferencias significativas entre las funciones estudiadas: superior proporción de diabéticos e inferior proporción de casos con hipercolesterolemia con SCORE. Framingham y REGICOR no presentaron diferencias notorias en la proporción de FRCV.

\section{DISCUSIÓN}

La ecuación calibrada REGICOR ${ }^{2}$ con el punto de corte $\geq 10 \%$ a los 10 años ha mos- trado unos buenos resultados en los aspectos de validez analizados. Ha presentado en relación a Framingham ${ }^{5}$ una proporción inferior, aunque no en exceso, de personas con RA, una buena correlación, una buena concordancia con el punto de corte $\geq 10 \%$ y un perfil de FRCV similar en los casos de RA. La ecuación de $\mathrm{SCORE}^{3}$ presentó unos valores de correlación y concordancia peores como era de esperar, puesto que REGICOR es la ecuación de Framingham pero calibrada, y se comportó de manera contradictoria al intentar extrapolar sus resultados a los pacientes hasta los 74 años: peor correlación pero mejor concordancia, que además tuvieron valores bastante inferiores en mujeres. La proporción de casos de RA fue superior a Framingham ${ }^{5}$ al extrapolar el riesgo hasta los 74 años e incluyó a más diabéticos y menos hipercolesterolémicos en los casos definidos como de RA.

Aunque el presente trabajo no es multicéntrico, la población de origen es bastante numerosa, heterogénea, y, de hecho, el tamaño muestral es superior al trabajo de Jones et $\mathrm{al}^{13}$ que sirvió para adoptar las recomendaciones británicas. La proporción de no respuestas fue inferior al $25 \%$, por lo que no creemos que se hayan introducido sesgos de selección. Aunque las funciones de Framingham permiten calcular el RCV a partir de los 30 años, se excluyó a los pacientes entre 30 y 34 por su bajo riesgo, de manera similar al grupo REGICOR ${ }^{2}$, facilitando 
además la comparabilidad con otros estu$\operatorname{dios}^{6}$

Nuestros pacientes presentaron (tabla 1) una mayor proporción de tabaquismo y diabetes mellitus y una menor proporción de hipertensos en varones respecto al estudio del grupo REGICOR ${ }^{6}$. Respecto al trabajo de Maiques Galán et $\mathrm{al}^{7}$ nuestros pacientes difirieron en edad (10 años más), mayor proporción de varones fumadores, inferior proporción de varones hipertensos y superior proporción de mujeres con hipercolesterolemia. Creemos por ello que el RCV de nuestros pacientes es similar al estudio del grupo REGICOR $^{6}$ y superior al trabajo de Maiques Galán et $\mathrm{al}^{7}$ y por ello el RCV medio con SCORE y Framingham fue superior al trabajo mencionado ${ }^{7}$, aunque los datos no son exactamente comparables al utilizarse en dicho estudio la función de Framingham de $1991^{4}$, además de otras diferencias.

Llama la atención que a pesar de ser inferior el RCV medio calculado con SCORE que el calculado con Framingham (tabla 2), la proporción de personas de RA (tabla 3 ) es sólo algo inferior con SCORE hasta los 65 años y es superior con SCORE hasta los 74 años (dobla la proporción respecto a SCORE hasta los 65 años), sobre todo en los diabéticos (tabla 4). En el estudio reseñado 7 la proporción de pacientes de RA fue inferior $(5,5 \%)$, pero se seleccionó a pacientes entre 30 y 75 años (SCORE contempla el cálculo entre 40 y 65), se asumió que el RCV entre 66 y 74 años era equivalente al de 65 años (en el artículo no se detallan los argumentos para realizar esta asunción) y se excluyó a los diabéticos, por lo que no es de extrañar el bajo RCV con SCORE en dicho estudio ${ }^{7}$.

La correlación de REGICOR ${ }^{2}$ con la ecuación de Framingham ${ }^{5}$ fue excelente (figura 1 ), con valores cercanos a 1 (correlación perfecta). Las dos líneas, prácticamente rectas, que se observan en la figura 1 corresponden a la correlación entre varones y mujeres (líneas superior e inferior, respectivamente). Estas cifras no son sorprendentes, puesto que la ecuación calibrada de REGICOR ${ }^{2}$ utiliza el mismo modelo y coeficientes beta que Framingham $^{5}$, procedimiento avalado por múltiples estudios ${ }^{14}$, que han demostrado que, en general, el riesgo relativo de los FRCV es similar de unos a otros países y lo que varía en realidad son los riesgos absolutos. Como era de esperar, la correlación (figuras 2 y 3 ) ha sido bastante peor con $\mathrm{SCORE}^{3}$ respecto a Framingham $^{5}$, especialmente en mujeres y si extrapolamos el RCV hasta los 74 años como han hecho otros autores ${ }^{7}$.

Respecto a la concordancia al catalogar a los pacientes de RA respecto a Framingham $^{5}$, el mejor valor fue con REGICOR ${ }^{2}$ con el punto de corte $\geq 10 \%$, con un valor de 0,83 . Con $\mathrm{SCORE}^{3}$ la concordancia fue bastante inferior $(0,61$ y 0,63$)$, sobre todo en las mujeres (cifras $<0,50$, indicativas de baja concordancia), como cabría esperar al ser Famingham y REGICOR la misma ecuación. En el estudio del grupo REGICOR ${ }^{6}$ con el punto de corte $\geq 10 \%$ a los 10 años la concordancia respecto a Framingham también fue excelente, con un valor de 0,88 (cifra obtenida a partir de los datos de dicho trabajo). En el estudio de Maiques Galán et $\mathrm{al}^{7}$ la concordancia de $\mathrm{SCORE}^{3}$ respecto a Framingham4 tampoco fue demasiado alta $(0,72)$. Por tanto no parece aconsejable utilizar las tablas de $\mathrm{SCORE}^{3}$ hasta los 74 años como han hecho otros autores ${ }^{7}$ hasta que no se haya demostrado su validez.

El perfil de los FRCV (tabla 7) mostró con SCORE una inferior proporción de pacientes con hipercolesterolemia y una superior proporción de diabetes mellitus. El trabajo de Maiques Galán et al $^{7}$ que también estudió esta cuestión, excluyó a los diabéticos y en él los pacientes de RA con SCORE tenían una proporción similar de pacientes con hipercolesterolemia y una superior proporción de pacientes con hipertensión. No obstante, la selección de los pacientes no fue aleatoria ${ }^{7}$, por lo que no se pueden descartar sesgos a este nivel. 
Puesto que está claro que hay que tratar con fármacos a los pacientes con hipertensión y diabetes mellitus que no se controlan con medidas higiénicas y dietéticas ${ }^{2}$, en la práctica real el RCV se puede disminuir básicamente mediante el uso de antiagregantes e hipolipemiantes (estatinas sobre todo). Utilizando REGICOR ${ }^{2}$ con el punto de corte del 10\% (el de mejor concordancia con Framingham) se trataría a una proporción inferior (pero no excesiva) de pacientes respecto a Framingham y utilizando SCORE hasta los 74 años aún se trataría a más pacientes que con Framingham, a pesar de que dicha función sólo predice mortalidad cardiovascu$\operatorname{lar}^{3}$. Con SCORE (tabla 7) se trataría con hipolipemiantes a pacientes de RA con una proporción inferior de casos con hipercolesterolemia, que es precisamente donde está más demostrada su eficacia de dichos fárma$\cos ^{15}$.

Estimar sólo el riesgo de mortalidad como hace $\mathrm{SCORE}^{3}$ supone en cierta medida desvirtuar la realidad de la práctica clínica, especialmente en atención primaria, en que la mayoría de acontecimientos cardiovasculares no son, afortunadamente, mortales. El otro gran inconveniente de $\mathrm{SCORE}^{3}$ es no disponer de tablas para diabéticos, población de elevado RCV en España16 y cuya prevalencia está aumentando en las últimas décadas $^{8,17}$. Por otro lado, la función REGICOR ${ }^{2}$ tiene como inconveniente estimar sólo el riesgo coronario, que aunque es una aproximación no puede considerase como un estimador ideal del riesgo cardiovascular. Jimeno Mollet et al ${ }^{18}$ señalan en su estudio con diabéticos que Framingham al 20\% sobreestima el RCV y REGICOR al 20\% lo infraestima, aunque la verdadera incidencia de cardiopatía isquémica está más próxima a REGICOR. Con el punto de corte $\geq 10 \%$ REGICOR consideraría a la diabetes entre la prevención primaria y la secundaria (tabla 4 ), coincidiendo con la tendencia actual ${ }^{19-20}$, mientras que Framingham y SCORE tienden a desplazar a los diabéticos hacia la prevención secundaria (tabla 4).
La publicación de las tablas del estudio DORICA $^{21}$, con una metodología similar a la ecuación calibrada de REGICOR, aunque con distinta selección de los pacientes al basarse en estudios transversales, supone una interesante aportación, pero ha complicado todavía más la crucial cuestión de que método de calculo del RCV es el más adecuado en España ${ }^{1}$. Aunque no hay estudios publicados que hayan demostrado la validez poblacional de $\mathrm{SCORE}^{3}$ ni de ninguna otra función, las Sociedades Europeas ${ }^{22}$ se han decantado por $\mathrm{SCORE}^{3}$ en sustitución de la ecuaciones clásicas de Framingham ${ }^{4,5}$. Asimismo, un grupo multidisciplinario español $^{23}$ también ha optado en fecha muy reciente por $\mathrm{SCORE}^{3}$, aunque señala que esta cuestión se puede revisar cuando se publiquen las validaciones de REGICOR y SCORE, como en el caso del estudio multicéntrico VERIFICA. Mientras tanto, el presente trabajo sugiere a partir de los parámetros de validez estudiados que la ecuación calibrada REGICOR ${ }^{2}$ es una buena herramienta para estimar el riesgo en un país de baja incidencia como el nuestro.

\section{Addendum}

Los firmantes del presente estudio no pertenecen a los grupos de trabajo REGICOR o SCORE. Los participantes no han sido incluidos en el estudio VERIFICA, promovido por la Fundación Jordi Gol i Gurina, el Institut Municipal de Investigació Médica de Barcelona y el Institut Català de la Salut.

\section{BIBLIOGRAFÍA}

1. Maiques Galan A. Valoración del riesgo cardiovascular. ¿Qué tabla utilizar? Aten Primaria 2003; 32: 586-89.

2. Marrugat J, Solanas P, D' Agostino R, Sullivan L, Ordovas J, Cordón F, et al. Estimación del riesgo coronario en España mediante la función de Framingham calibrada. Rev Esp Cardiol 2003; 56: 253-61. 
3. Conroy RM, Pyörälä K, Fitzgerald AP, Sans S, Menotti A, De Backer G, et al. Estimation of tenyear risk of fatal cardiovascular disease in Europe: the SCORE project. Eur Heart J 2003; 24: 987 1003.

4. Anderson KM, Wilson PWF, Odell PM, Kannel WB. Un update coronary risk profile. A statement for health professionals. Circulation 1991; 83: 356-2.

5. Wilson PWF, D'Agostino RB, Levy D, Belanger AM, Silbershatz H, Kannel WB. Prediction of coronary heart disease using risk factor categories. Circulation 1998; 97; 1837-47.

6. Ramos R, Solanas P, Cordón F, Rohlfs I, Elosua R, Sala J, et al. Comparación de la función de Framingham original y la calibrada del REGICOR en la predicción del riesgo coronario poblacional. Med Clin (Barc) 2003; 121: 521-6.

7. Maiques Galán A, Antón García F, Franch Taix M, Albert Ros X, Aleixandre Martí E, Collado Gil A. Riesgo cardiovascular del SCORE comparado con el de Framingham. Consecuencias del cambio propuesto por las Sociedades Europeas. Med Clin (Barc) 2004; 123: 681-5.

8. Baena Díez JM, del Val García JL, Tomàs Pelegrina J, Martínez Martínez JL, Martín Peñacoba R, González Tejón I, et al. Epidemiología de las enfermedades cardiovasculares y factores de riesgo en atención primaria. Rev Esp Cardiol 2005; 58: 367-73.

9. Baena Díez JM, Álvarez Pérez B, Piñol Forcadell P, Martín Peñacoba R, Nicolau Sabaté M, Altès Boronat A. Asociación entre la agrupación (clustering) de factores de riesgo cardiovascular y el riesgo de enfermedad cardiovascular. Rev Esp Salud Pública 2002; 76: 7-15.

10. Córdoba García R, Ortega Sánchez-Pinilla R, Cabezas Peña C, Forés García D, Nebot Adell M. Recomendaciones sobre el estilo de vida. Aten Primaria 1999; 24 (supl. 1): 118-32.

11. Programa de Actividades preventivas y de Promoción de la Salud. Guía de Prevención Cardiovascular. Madrid: Sociedad Española de Medicina Familiar y Comunitaria, 1996; 18-36.

12. The Expert Committee on the diagnosis and Classification of Diabetes Mellitus: Report of the Committee on the diagnosis and Classification of Diabetes Mellitus. Diabetes Care 1997; 20:1183-97.

13. Jones AF, Walker J, Jewkes C, Game FL, Barlett WA, Marshall T, et al. Comparative accuracy of cardiovascular risk prediction methods in primary care patiens. Heart 2001; 85: 37-43.
14. D'Agostino RB, Grundy S, Sullivan LM, Wilson P. Validation of the Framingham Coronary Heart Disease Prediction Scores: Results of a Multiple Ethnic Groups Investigation. JAMA 2001; 286: 180-7.

15. Pignone M, Phillips C, Mulrow C. Use of lipid lowering drugs for primary prevention of coronary heart disease: meta-analisys of randomised trials. BMJ 2000; 321: 1-5.

16. Baena Díez JM, González Tejón I, Pomares Sajkiewick M, Rovira España M, Martínez Martínez JL, Tomàs Pelegrina J. Episodios cardiovasculares asociados a las nuevas categorías diagnósticas de la diabetes mellitus propuestas por la Asociación Americana de Diabetes. Aten Primaria 2002; 29: 343-7.

17. Mokdad AH, Bowman BA, Ford ES, Vinicor F, Marks JS, Koplan JP. The continuing epidemics of obesity and diabetes in the United States. JAMA 2001; 286: 1195-200.

18. Jimeno Mollet J, Molist Brunet N, Franch Nadal J, Serrano Borraz V, Serrano Barragán L, Gracia Jiménez R. Variabilidad en la estimación del riesgo coronario en la diabetes mellitus tipo 2. Aten Primaria 2005; 35: 30-6.

19. Evans JMM, Wang J, Morris AD. Comparison of cardiovascular risk between patiens with type 2 diabetes and those who had had a myocardial infarction: cross sectional and cohort studies. BMJ 2002; 324:939-44.

20. Lee CD, Folsom AR, Pankow JS, Brancati FL. Atherosclerosis Risk in Communities (ARIC) Study Investigators. Cardiovascular events in diabetic and nondiabetic adults with or without history of myocardial infarction. Circulation 2004; 109 : 855-60.

21. Aranceta J, Pérez Rodrigo C, Foz Sala M, Mantilla T, Serra Majem L, Moreno B, et al. Tablas de evaluación del riesgo coronario adaptadas a la población española. Estudio DORICA. Med Clin (Barc) 2004; 123: 686-91.

22. De Backer G, Ambrosioni E, Borch-Johsen K, Brotons C, Cifkova R, Dallongeville J, et al. Executive summary. European guidelines on cardiovascular disease prevention in clinical practice. Eur Heart $\mathbf{J}$ 2003; 24: 1601-10.

23. Comité Español Interdisciplinario para la Prevención Cardiovascular. Adaptación española de la Guía Europea de Prevención Cardiovascular. Rev Esp Salud Publica 2004; 78: 435-8. 